\title{
Sleep/Wake Scheduling for Target Coverage Problem in Wireless Sensor Networks
}

\author{
Manirajan.R ${ }^{1}$, Sathishkumar.K.R ${ }^{2}$ \\ PG scholar, (M.E-Communication \& Networking) Mahendra Engineering College, Namakkal, India ${ }^{1}$ \\ Assistant Professor, CSE, Mahendra Engineering College, Namakkal, India ${ }^{2}$
}

\begin{abstract}
Network lifetime plays an integral role in setting up an efficient wireless sensor network. The objective is in twofold. The first one is to deploy sensor nodes at optimal locations such that the theoretically computed network lifetime is maximum. The second is to schedule these sensor nodes such that the network attains the maximum lifetime. Thus, the overall objective is to identify optimal deployment locations of the given sensor nodes with a pre-specified sensing range, and to schedule them such that the network lifetime is maximum with the required coverage level. Since the upper bound of the network lifetime for a given network can be computed mathematically, use this knowledge to compute locations of deployment such that the network lifetime is maximum. Further, the nodes are scheduled to achieve this upper bound.

In this proposed system uses Sleep-wake scheduling is an effective mechanism to prolong the lifetime of energyconstrained wireless sensor networks. However, sleep-wake scheduling could result in substantial delays because a transmitting node needs to wait for its next-hop relay node to wake up. An interesting line of work attempts to reduce these delays by developing "anycast"-based packet forwarding schemes, where each node opportunistically forwards a packet to the first neighboring node that wakes up among multiple candidate nodes.

It develops an anycast packet-forwarding scheme to reduce the event-reporting delay and to prolong the lifetime of wireless sensor networks employing asynchronous sleep-wake scheduling. Specifically, studies two optimization problems. First, when the wake-up rates of the sensor nodes are given, develop an efficient and distributed algorithm to minimize the expected event-reporting delay from all sensor nodes to the sink. Second, using a specific definition of the network lifetime, studies the lifetime-maximization problem to optimally control the sleep-wake scheduling policy and the any cast policy in order to maximize the network lifetime subject to an upper limit on the expected end-to-end delay.
\end{abstract}

Keywords: Sleep/Wake scheduling, Energy Consumption, Improve the network life time, Minimizing Delay, Wireless sensor Network

\section{INTRODUCTION}

WIRELESS Sensor Networks (WSNs) are important for many applications such as military sensing, physical security, air traffic control, traffic surveillance, video surveillance, industrial and manufacturing automation, environment monitoring, and building and structural monitoring. Network lifetime (defined as the time instant from which the network starts functioning to the time instant where the desired coverage criterion is not satisfied) is a crucial factor that determines the efficiency of a wireless sensor network. Energy usage should be curbed to achieve enhanced lifetime. This is because sensor nodes are battery powered and cannot be easily recharged or replaced.

Coverage in a WSN needs to guarantee that the region is monitored with the required degree of reliability. Locations of sensor nodes constitute the basic input for the algorithms that examine coverage of the network. Coverage problems can be broadly classified as area coverage problem and target coverage problem. Area coverage focuses on monitoring the entire region of interest, whereas target coverage concerns monitoring only certain specific points in a given region. Target coverage can be categorized as simple coverage, k-coverage and $\mathbf{Q}$ coverage.
There are two types of sensor node deployments: random deployment and deterministic deployment. Random deployment is suitable for applications where the details of the regions are not known, or regions are inaccessible. An example of random deployment of sensor nodes would be in battlefield surveillance. In such a deployment, the most common way of extending the network lifetime is by scheduling the sensor nodes such that only a subset of sensor nodes that is enough to satisfy coverage requirement need to be active at a time.

In deterministic deployment, the details of the region will be known apriori and since a provision of deploying nodes at specific locations prevail, there exists two ways by which network lifetime can be maximized. One is at deployment phase and the other is at scheduling phase. Given a region with targets being monitored by sensor nodes, the upper bound of network lifetime can be mathematically computed. This information can be used for computing locations which would be appropriate for coverage to be satisfied as well as network lifetime to be maximum. Once the deployment locations are computed, sensor nodes can be scheduled to achieve the optimum lifetime. Sensor deployment and scheduling in this way contributes equally to extend the network lifetime. Hence 
the problem can be summarized as: Given some sensor nodes that can be deterministically deployed, where to deploy them and how to schedule them so as to achieve the required target coverage level and maximize the network lifetime?

The main objective of proposed system, first study how to optimize the any cast forwarding schemes for minimizing the expected packet-delivery delays from the sensor nodes to the sink. Based on this result, then provide a solution to the joint control problem of how to optimally control the system parameters of the sleep-wake scheduling protocol and the any cast packet-forwarding protocol to maximize the network lifetime, subject to a constraint on the expected end-to-end packet-delivery delay.

\section{RELATED WORK}

Sensor Networks: Evolution, Opportunities, and Challenges concludes by presenting some recent research results in sensor network algorithms, including localized algorithms and directed diffusion, distributed tracking in wireless ad hoc networks, and distributed classification using local agents. The disadvantage is high cost and reliability is less. However, More reliable wireless communication and low-cost manufacturing have resulted in small, inexpensive, and powerful sensors with embedded processing and wireless networking capability.

A survey on sensor localization presents a comprehensive survey on sensor localization in WSNs covering motivations, problem formulations, solution approaches and performance summary. Future research issues will also be discussed. Algorithms used are Simple anchor-free localization algorithm, Distributed Algorithm, Centralized algorithm. The disadvantages localization problem is formulated as a pattern recognition problem with its kernel matrix being the signal strength matrix. And motivates the readers by identifying the basic problems in deployment, data gathering, and optimal resource usages; and thereby examine how localization becomes critical to the applications of WSNs. An important problem in the deployment of WSNs is the coverage. A coverage model of sensor nodes would depend on the distance between the point of interest and the closest node. However the advantages of LR protocols include better scalability and less overhead caused by dynamic changes in topology. The use of mobile reference nodes in localization is advantageous, because it provides additional measurements on spatial relationships along their corresponding trajectories.

The Coverage Problem in a Wireless Sensor Network applications of the result include: (i) positioning applications, (ii) situations which require stronger environmental monitoring capability, and (iii) scenarios which impose more stringent fault-tolerant capability. The algorithm used are polynomial-time algorithms, optimum algorithms, density control algorithm. The disadvantages: One fundamental issue in sensor networks is the coverage problem, which reflects how well a sensor network is monitored or tracked by sensors. However the sensing range of each sensor can be a unit disk or a non-unit disk. The solution can be easily translated to a distributed protocol where each sensor only needs to collect local information to make its decision. Instead of determining the coverage of each location, this approach tries to look at how the perimeter of each sensor's sensing range is covered, thus leading to an efficient polynomial time algorithm. As long as the perimeters of sensors are sufficiently covered, the whole area is sufficiently covered.

A survey: algorithms simulating bee swarm intelligence says that intelligent behaviors of bee swarm have inspired the researchers especially during the last decade to develop new algorithms. This work presents a survey of the algorithms described based on the intelligence in bee swarms and their applications. Algorithm used queen-bee evolution algorithm, genetic algorithm. The disadvantages are economic power dispatch problem which is formulated as a nonlinear constrained complex optimization problem the queen-bee crossover in GA to make the procedure more efficient for the label-constrained minimum spanning tree problem. The problem of pattern formation on a grid, for a group of identical autonomous robotic agents by the communication between the agents. However, Swarms use their environment and resources effectively by collective intelligence. Self-organization is a key feature of a swarm system which results global level (macroscopic level) response by means of low level interactions (microscopic level).

On the performance of artificial bee colony (ABC) algorithm says that the performance of the $\mathrm{ABC}$ algorithm has been compared with that of differential evolution, particle swarm optimization and evolutionary algorithm for multi-dimensional and multimodal numeric problems. The algorithms used are Artificial bee colony (ABC) algorithm, Evolutionary algorithms (EA), DE algorithm, genetic algorithm. The main disadvantage of poor local search ability of genetic algorithm (GA) PSO consists of a swarm of particles moving in a search space of possible solutions for a problem. The advantage of $\mathrm{ABC}$ algorithm performs better than the mentioned algorithms and can be efficiently employed to solve the multimodal engineering problems with high dimensionality.

Designing k-coverage schedules in wireless sensor networks investigates the sensor scheduling for k-coverage (ssc) problem which requires to efficiently scheduling the sensors, such that the monitored area can be k-covered throughout the whole network lifetime with the purpose of maximizing network lifetime. the ssc problem is np-hard and propose two heuristic algorithms under different scenarios. in addition, develop a guideline for users to better design a sensor deployment plan to save energy by employing a density control scheme. heuristic algorithms, greedy- selection (gs) algorithm are used. The disadvantage is the problem with more constraints, such as connectivity and communication range, bandwidth limitation, transmission delay requirement and etc. however, advantage is the purpose of maximizing the network lifetime.

Lightweight deployment-aware scheduling for wireless sensor networks says that the problem of estimating 
redundant sensing areas among neighbouring wireless sensors is analyzed. It presents simple methods to estimate the degree of redundancy without the knowledge of location or directional information. also provide tight upper and lower bounds on the probability of complete redundancy and on the average partial redundancy. The algorithm is deployment-aware scheduling (ldas) algorithm, scheduling algorithms. The disadvantages are sensor networks will still be effective for most applications as long as a reasonable coverage is maintained. Because of this reason, it is critical to provide good scheduling algorithms to turn off sensors without degrading sensing coverage significantly in a statistical sense. However, advantage is a mathematical model to describe the redundancy in randomly deployed sensor networks.

Obstacle-resistant deployment algorithms for wireless sensor networks says that the efficient obstacle-resistant robot deployment (orrd) algorithm, which involves the design of a node placement policy, a serpentine movement policy, and obstacle-handling rules. performance analysis of the proposed orrd is given in terms of the number of required sensors for an environment containing several irregular obstacles. simulation results revealed that the proposed orrd overcomes the unpredicted obstacles and deploys fewer static sensors but achieves higher coverage percentages compared to existing deployment algorithms. The algorithm used obstacle-resistant robot deployment (orrd) algorithm. The disadvantage is global information is not possible in an unexplored region, the developed mechanism could only be used for limited applications. The advantage is the proposed orrd overcomes the unpredicted obstacles and deploys fewer static sensors but achieves higher coverage percentages compared to existing deployment algorithms.

Quality of deployment in surveillance wireless sensor networks proposes several quality measures, which indicate if the deployment provides sufficient coverage, or whether redeployment is required or not. the terrain is modeled as a grid and the placement of the sensors is uniformly distributed. neyman-pearson detection is utilized to determine the effects of false-alarm and signal characteristics on the measures. Algorithms used are labeling algorithm, dijkstra's algorithm. The disadvantage considering the surveillance applications, the geographical properties of the field, such as the altitude, may affect the deployment, as well. if the field is a narrow canyon, the bottom locations will be occupied by more sensors. these problems require three-dimensional field models and analysis of non-uniform deployment. However, advantage is the coordinates of the sensor positions are assumed to be drawn from independent uniform distributions so that there is no bias in any direction.

Deploying wireless sensors to achieve both coverage and connectivity says that an optimal deployment pattern to achieve both full coverage and 2-connectivity, and prove its optimality for all values of $\mathrm{rc} / \mathrm{rs}$, where $\mathrm{rc}$ is the communication radius, and $\mathrm{rs}$ is the sensing radius. The algorithm used heuristic algorithm. The disadvantage with the emergence of wireless sensor networks, however, it is now no longer enough to consider coverage alone when deploying a wireless sensor network; connectivity must also be considered. While moderate loss in coverage can be tolerated by applications of wireless sensor networks, loss in connectivity can be fatal. However, advantage of an optimal deployment pattern to achieve both full coverage and 2-connectivity, and prove its optimality for all values.

\section{PROPOSED SYSTEM}

Sleep-wake scheduling is an effective mechanism to prolong the lifetime of energy-constrained wireless sensor networks. is an effective Mechanism to prolong the lifetime of these energy-constrained wireless sensor networks. However, sleep-wake scheduling could result in substantial delays because a transmitting node needs to wait for its next-hop relay node to wake up. An interesting line of work attempts to reduce these delays by developing "anycast"-based packet forwarding schemes, where each node opportunistically forwards a packet to the first neighboring node that wakes up among multiple candidate nodes.

In proposed system develops an anycast packetforwarding scheme to reduce the event-reporting delay and to prolong the lifetime of wireless sensor networks employing asynchronous sleep-wake scheduling. Specifically, studies two optimization problems. First, when the wake-up rates of the sensor nodes are given, Develop an efficient and distributed algorithm to minimize the expected event-reporting delay from all sensor nodes to the sink.

Second, using a specific definition of the network lifetime, study the lifetime-maximization problem to optimally control the sleep-wake scheduling policy and the any cast policy in order to maximize the network lifetime subject to an upper limit on the expected end-to-end delay. The advantages are sleep/wake scheduling for low duty-cycle sensor networks, consider synchronization error, achieve given capture probability threshold with min energy consumption.

Any cast objectives and performance metrics defines the performance objectives of the any cast policy and the sleep-wake scheduling policy that intend to optimize. It remind the readers that, although the sleep-wake patterns and the any cast forwarding policy are applied in the operation phase of the network, their control parameters are optimized in the configuration phase.

\section{1) End-to-End Delay:}

It defines the end-to-end delay as the delay from the time when an event occurs to the time when the first packet due to this event is received at the sink. Motivate this performance objective as follows: For applications where each event only generates one packet, the above definition clearly captures the delay of reporting the event information.

\section{2) Network Lifetime:}

Introduces the second performance metric, the network lifetime, and the corresponding lifetime-maximization problem (subject to delay constraints). Minimization of end-to-end delays: The delay-minimization problem is an 
instance of the stochastic shortest path (SSP) problem, where the sensor node that holds the packet corresponds to the "state," and the delay corresponds to the "cost" to be minimized. The sink then corresponds to the terminal state, where the cost (delay) is not incurred anymore. Let be the sequence of nodes hat successively relay the packet from the source node to ink node. Note that the sequence is random because at each hop, the first node in the forwarding set that wakes up will be chosen as a next-hop node. Globally optimal forwarding and priority matrices develop an algorithm computing the globally optimal forwarding and priority matrices for given $p$. This algorithm has the flavor of the distributed Bellman-Ford's algorithm for finding the shortest paths. At each iteration, each node uses the delay estimates from the previous iteration to update the forwarding set and the priority assignment

\section{SYSTEM ARCHITECTURE}

A system architecture or systems architecture is the conceptual design that defines the structure and/or behavior of a system. An architecture description is a formal description of a system, organized in a way that supports reasoning about the structural properties of the system. It defines the system components or building blocks and provides a plan from which products can be procured, and systems developed, that will work together to implement the overall system. This may enable one to manage investment in a way that meets business needs.

The fundamental organization of a system, embodied in its components, their relationships to each other and the environment, and the principles governing its design and evolution. The composite of the design architectures for products and their life cycle processes. A representation of a system in which there is a mapping of functionality onto hardware and software components, a mapping of the software architecture onto the hardware architecture, and human interaction with these components. An allocated arrangement of physical elements which provides the design solution for a consumer product or life-cycle process intended to satisfy the requirements of the functional architecture and the requirements baseline. Architecture is the most important, pervasive, top-level, strategic inventions, decisions, and their associated rationales about the overall structure and associated characteristics and behavior.

\section{PROJECT DESCRIPTION SOURCES}

The main goal of the single source that sends out eventreporting packets to the sink. In such systems, there are four main sources of energy consumption: energy required keeping the communication radios on; energy required for the transmission and reception of control packets; energy required to keep sensors on; and energy required for data transmission and reception. The fraction of total energy consumption for data transmission and reception is relatively small in these systems because events occur so rarely.

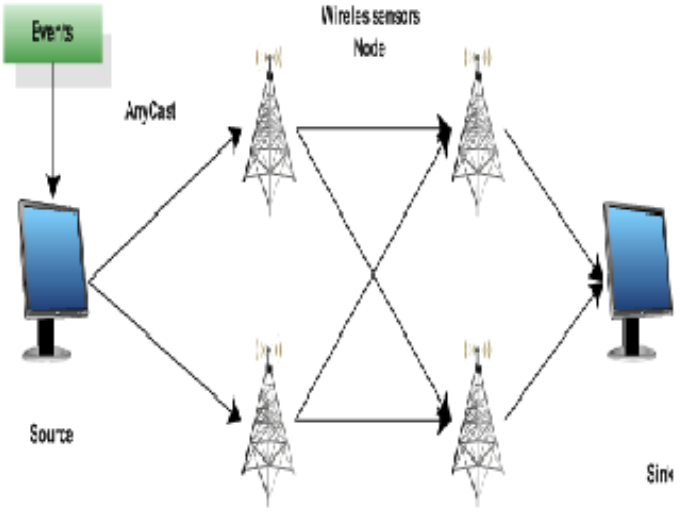

\section{MAXIMIZING LIFETIME}

In such systems, there are four main sources of energy consumption: energy required to keep the communication radios on; energy required for the transmission and reception of control packets;

Energy required keeping sensors on; and energy required for data transmission and reception. The fraction of total energy consumption for data transmission and reception is relatively small in these systems because events occur so rarely.

The energy required to sense events is usually a constant and cannot be controlled. Hence, the energy expended to keep the communication system on (for listening to the medium and for control packets) is the dominant component of energy consumption, which can be controlled to extend the network lifetime. Thus, sleepwake scheduling becomes an effective mechanism to prolong the lifetime of energy-constrained event-driven sensor networks. By putting nodes to sleep when there are no events, the energy consumption of the sensor nodes can be significantly reduced.

\section{MINIMIZING DELAY}

Prior work in the literature has proposed the use of any cast packet-forwarding schemes (also called opportunistic forwarding schemes) to reduce this event reporting delay. Under traditional packet-forwarding schemes, every node has one designated next-hop relaying node in the neighborhood, and it has to wait for the next-hop node to wake up when it needs to forward a packet.

In contrast, under anycast packet-forwarding schemes, each node has multiple next-hop relaying nodes in a candidate set (call this set the forwarding set) and forwards the packet to the first node that wakes up in the forwarding set. It is easy to see that, compared to the basic scheme in; anycast clearly reduces the expected one-hop delay. For example, assuming that there are nodes in the forwarding set, and that each node wakes up independently according to the Poisson process with the same rate, then anycast can result in a-fold reduction in the expected one-hop delay.

\section{ANY CAST FORWARDING}

An interesting line of work attempts to reduce these delays by developing "any cast"-based packet forwarding schemes, where each node opportunistically forwards a packet to the first neighboring node that wakes up among 
multiple candidate nodes. In the proposed system first study how to optimize the any cast forwarding schemes for minimizing the expected packet-delivery delays from the sensor nodes to the sink. Any cast clearly reduces the expected one-hop delay.

\section{SLEEP-WAKE SCHEDULING}

Sleep-wake scheduling is an effective mechanism to prolong the lifetime of these energy-constrained wireless sensor networks. The energy required to sense events is usually a constant and cannot be controlled. Hence, the energy expended to keep the communication system on (for listening to the medium and for control packets) is the dominant component of energy consumption, which can be controlled to extend the network lifetime. Thus, sleepwake scheduling becomes an effective mechanism to prolong the lifetime of energy-constrained event-driven sensor networks. By putting nodes to sleep when there are no events, the energy consumption of the sensor nodes can be significantly reduced. In proposed system proposes asynchronous sleep-wake scheduling protocols. In these protocols, each node wakes up independently of neighboring nodes in order to save energy.

The proposed system assumes that the sensor network employs asynchronous sleep-wake scheduling to improve energy efficiency, and nodes choose the next-hop node and forward the packet to the chosen node using the following basic sleep-wake scheduling protocol. The advantage of Poisson sleep-wake scheduling is that, due to its memory less property, sensor nodes are able to use a time-invariant optimal policy to maximize the network lifetime while the analysis focuses on the case when the wake-up times follow a Poisson process.

\section{SINK}

Sink is a base station, this is a final stage, and the events are received from different wireless sensor node by this stage. To optimize the any cast forwarding schemes for minimizing the expected packet-delivery delays from the sensor nodes to the sink. If a node detects an event, the node packs the event information into a packet and delivers the packet to a sink via multi hop relaying. Assume that every node has at least one such multi hop path to the sink. Also assume that there is a single sink.

\section{DATA FLOW DIAGRAM}

A data-flow diagram (DFD) is a graphical representation of the "flow" of data through an information system. DFDs can also be used for the visualization of data processing (structured design). On a DFD, data items flow from an external data source or an internal data store to an internal data store or an external data sink, via an internal process.

A DFD provides no information about the timing or ordering of processes, or about whether processes will operate in sequence or in parallel. It is therefore quite different from a flowchart, which shows the flow of control through an algorithm, allowing a reader to determine what operations will be performed, in what order, and under what circumstances, but not what kinds of data will be input to and output from the system, nor where the data will come from and go to, nor where the data will be stored (all of which are shown on a DFD).

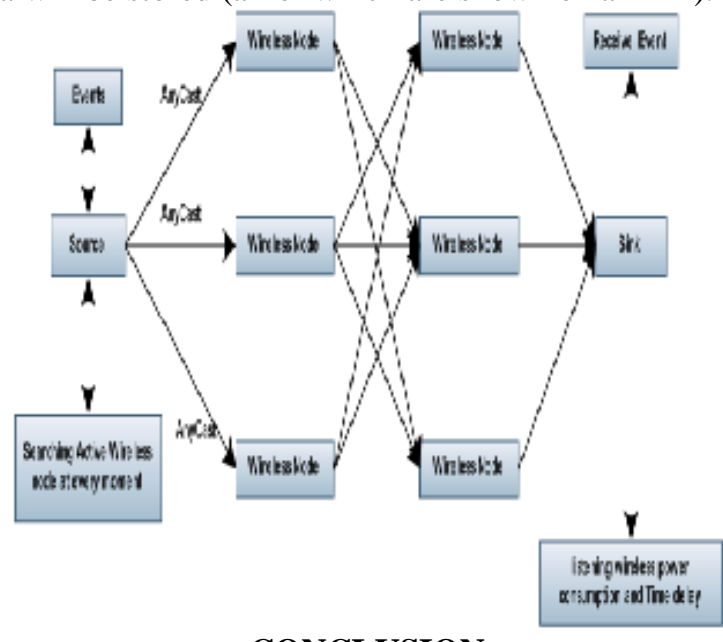

\section{CONCLUSION}

In this, develop an any cast packet-forwarding scheme to reduce the event-reporting delay and to prolong the lifetime of wireless sensor networks employing asynchronous sleep-wake scheduling. Specifically, studies two optimization problems. First, when the wake-up rates of the sensor nodes are given, develop an efficient and distributed algorithm to minimize the expected eventreporting delay from all sensor nodes to the sink. Second, using a specific definition of the network lifetime and study the lifetime-maximization problem to optimally control the sleep-wake scheduling policy and the any cast policy in order to maximize the network lifetime subject to an upper limit on the expected end-to-end delay. The numerical results suggest that the proposed solution can substantially outperform prior heuristic solutions in the literature under practical scenarios where there are obstructions in the coverage area of the wireless sensor network. For future work, plan to generalize solution to take into account non-Poisson wake-up processes and other lifetime definitions.

\section{REFERENCES}

C.-Y. Chong and S. Kumar, "Sensor networks: Evolution, opportunities, and challenges," Proc. IEEE, vol. 91, no. 8, pp. 1247-1256, Aug. 2003

2. J. Wang, R. Ghosh, and S. Das, "A survey on sensor localization," J. Control Theory Appl., vol. 8, no. 1, pp. 2-11, 2010.

C.-F. Huang and Y.-C. Tseng, "The coverage problem in a wireless sensor network," in Proc. 2nd ACM Int. Conf. Wireless Sensor Netw. Appl., 2003, pp. 115-121.

4. D. Karaboga and B. Akay, "A survey: Algorithms simulating bee swarm intelligence," Artif. Intell. Rev., vol. 31, nos. 1-4, pp. 61-85, 2009

5. D. Karaboga and B. Basturk, "On the performance of artificial bee colony (ABC) algorithm," Appl. Soft Comput., vol. 8, pp. 687-697, Jan. 2008.

6. Y. $\mathrm{Li}$ and $\mathrm{S}$. Gao, "Designing k-coverage schedules in wireless sensor networks," J. Combinat. Opt., vol. 15, no. 2, pp. 127-146, 2008.

7. K. Wu, Y. Gao, F. Li, and Y. Xiao, "Lightweight deploymentaware scheduling for wireless sensor networks," Mobile Netw. Appl., vol. 10, pp. 837-852, Dec. 2005.

8. C.-Y. Chang, C.-T. Chang, Y.-C. Chen, and H.-R. Chang, "Obstacle resistant deployment algorithms for wireless sensor networks," IEEE Trans. Veh. Technol., vol. 58, no. 6, pp. 29252941, Jul. 2009. 
9. E. Onur, C. Ersoy, and H. Deliç, "Quality of deployment in surveillance wireless sensor networks," Int. J. Wireless Inform. Netw., vol. 12, no. 1, pp. 61-67, 2005.

10. X. Bai, S. Kumar, D. Xuan, Z. Yun, and T. H. Lai, "Deploying wireless sensors to achieve both coverage and connectivity," in Proc. 7th ACM Int. Symp. Mobile Ad Hoc Netw. Comput., 2006, pp. 131-142. X. Bai, Z. Yun, D. Xuan, T. Lai, and W. Jia, "Optimal patterns for four-connectivity and full coverage in wireless sensor networks," IEEE Trans. Mobile Comput., vol. 9, no. 3, pp. 435448, Mar. 2010.

11. G. Tan, S. Jarvis, and A.-M. Kermarrec, "Connectivity-guaranteed and obstacle-adaptive deployment schemes for mobile sensor networks," in Proc. 28th Int. Conf. Distrib. Comput. Syst., Jun. 2008, pp. 429-437. 